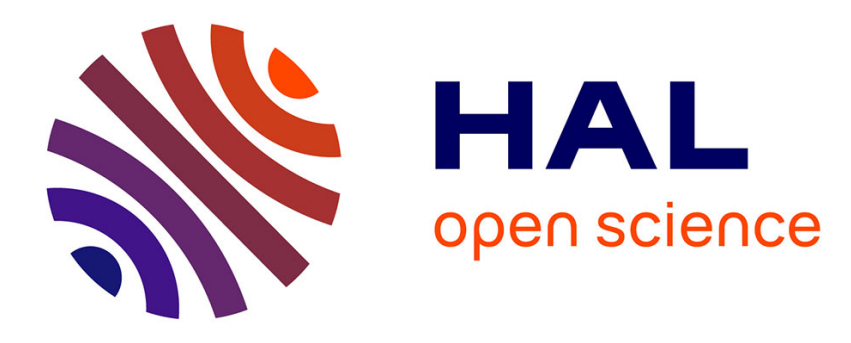

\title{
Use Cases of Pervasive Artificial Intelligence for Smart Cities Challenges
}

\author{
Julien Nigon, Estèle Glize, David Dupas, Fabrice Crasnier, Jérémy Boes
}

\section{To cite this version:}

Julien Nigon, Estèle Glize, David Dupas, Fabrice Crasnier, Jérémy Boes. Use Cases of Pervasive Artificial Intelligence for Smart Cities Challenges. IEEE Workshop on Smart and Sustainable City (WSSC 2016) associated to the International Conference IEEE UIC 2016, Jul 2016, Toulouse, France. pp. 1021-1027. hal-01484992

\section{HAL Id: hal-01484992 https://hal.science/hal-01484992}

Submitted on 8 Mar 2017

HAL is a multi-disciplinary open access archive for the deposit and dissemination of scientific research documents, whether they are published or not. The documents may come from teaching and research institutions in France or abroad, or from public or private research centers.
L'archive ouverte pluridisciplinaire HAL, est destinée au dépôt et à la diffusion de documents scientifiques de niveau recherche, publiés ou non, émanant des établissements d'enseignement et de recherche français ou étrangers, des laboratoires publics ou privés. 


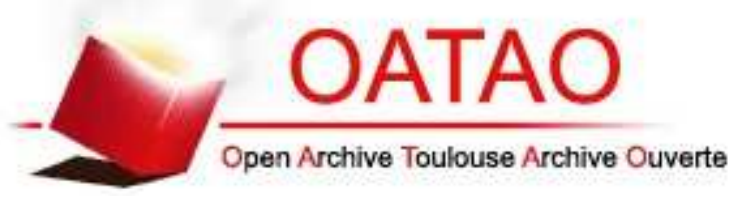

\section{Open Archive TOULOUSE Archive Ouverte (OATAO)}

OATAO is an open access repository that collects the work of Toulouse researchers and makes it freely available over the web where possible.

This is an author-deposited version published in : http://oatao.univ-toulouse.fr/ Eprints ID : 17122

The contribution was presented at WSSC 2016:

https://www.irit.fr/ Georges.Da-Costa/wssc/

To cite this version : Nigon, Julien and Glize, Estèle and Dupas, David and Crasnier, Fabrice and Boes, Jérémy Use Cases of Pervasive Artificial Intelligence for Smart Cities Challenges. (2016) In: IEEE Workshop on Smart and Sustainable City (WSSC 2016) associated to the International Conference IEEE UIC 2016, 18 July 2016 (Toulouse, France).

Any correspondence concerning this service should be sent to the repository administrator: staff-oatao@listes-diff.inp-toulouse.fr 


\title{
Use Cases of Pervasive Artificial Intelligence for Smart Cities Challenges
}

\author{
Julien Nigon ${ }^{1}$, Estèle Glize ${ }^{2}$, David Dupas ${ }^{1}$, Fabrice Crasnier $^{1}$, Jérémy Boes ${ }^{1}$
}

\begin{abstract}
Software engineering has been historically topdown. From a fully specified problem, a software engineer needs to detail each step of the resolution to get a solution. The resulting program will be functionally adequate as long as its execution environment complies with the original specifications. With their large amount of data and their ever changing multi-level dynamics, smart cities are too complex for a topdown approach. They prompt the need for a paradigm shift in computer science. Programs should be able to self-adapt on the fly, to handle unspecified events, and to efficiently deal with tremendous amount of data. To this end, bottom-up approach should become the norm. Machine learning is a first step, and distributed computing helps. Multi-Agent Systems (MAS) can combine machine learning and distributed computing and may be easily designed with a bottom-up approach. This paper explores how MASs can answer challenges at various levels of smart cities, from sensors networks to ambient intelligence.
\end{abstract}

\section{INTRODUCTION}

Nowadays, only $2 \%$ of the Earth's surface is occupied by cities. In 2050 , they will have $70 \%$ of the world population and they will release more than $80 \%$ of $\mathrm{CO} 2$ emissions. One of the main challenge for the smart city is to optimize the activities and the services it offers, while supervising the energies, and in maintaining a good quality of life for people who stay at the center of the smart cities concerns. To customize and optimize the inhabitant's environment, it is necessary to equip it with tools, mainly technological, which provide information about the environment. If Internet of Things is required to manage efficiently and relevantly a city, this is not sufficient. We present in this paper an Artificial Intelligence approach which can improve the decision-making for energy consumption and human well-being.

Section II introduces the definition of a smart city and states the challenges relative to a specific thematic, energy. Section III introduce AMOEBA, a multi-agent AI system designed to understand and manage pervasive systems which self-adapt in real-time, thus requiring no explicit control. Sections IV to VI present use cases of artificial intelligence at three different scales in the city of the future:

- A city-scale application is the forecast of renewable energy production in order to optimize energy management in the city. Section IV presents how AMOEBA learns a model of wind power production.

${ }^{1}$ Team SMAC, IRIT, Universitée Fédérale de Toulouse, 118 rte de Narbonne, 31062 Toulouse Cedex, France julien.nigon at irit. fr

$2 *$ SWIFT, 26/28 rue Marie Magné 31300 Toulouse, France estele.meteoswift at gmail.com
- At building-scale, section $\mathrm{V}$ gives results on automatic detection of singularities in order to improve the energy performance of buildings.

- At an individual scale, section VI presents the architecture AMOEBA architecture allow to understand what are the conditions of the well-being of a human in his daily life.

\section{SMART CITY}

Smart cities are a hot topic, very cross-disciplinary and consequently studied by many authors in various disciplines. Therefore, many definitions have been proposed, some with more emphasis on performance, or governance, or technology... [10]. Nevertheless, it is generally accepted that smart cities are using a large number of sensors and data sources, and although it is not the only constitutive part of a smart city it is to this aspect that we are interested in this paper.

The development of smart cities involves many areas of research to define the best ways to deal with and manage energy, mobility, health governance, etc. Nevertheless, one of the major differences between a smart city and a "conventional" city smartly designed is the extensive use of IT and communication technologies. This difference significantly alters the way in which we can use the available data to design the city of the future. Indeed, businesses and governments have regularly collected large amounts of data about cities, but these data sets are often characterized by a relatively small number of variables, low continuity in time, and are often in limited access [7]. Most of the time, conventional cities are characterized by data scarcity.

On the other hand, the proliferation of inexpensive sensors offers the perspective of having a continuous huge amount of heterogeneous data, with autonomous and distributed sources, also known as big data [14]. Given this trend, smart cities will be characterized by their ability to generate big data, unlike conventional cities. Issues related to the analysis of these data then change completely. Where the scarcity and irregularity of data were among the main issues to take into account, the main challenges become, for smart cities, to be able to process extensive data, sometimes containing useless or redundant sources, and to be able to extract useful information and correlations in real time.

Real time is indeed a really important feature for any system trying to handle data from smart city. For example, if data is used to improve transportation by advising travelers of the best path to choose, according to current flow of people, the analysis realized by the system must be able to provide a rapid solution [2]. Even after few minutes, the data may 
have changed dramatically. The analysis time must remain far below these time scales.

In this context, it is difficult to develop algorithms to establish relevant correlations in this huge stream of data. The difficulty increases even more when it comes to be able to process the data indifferently for each relevant field. Especially in the context of a smart city, where these fields are numerous (energy, transport, thermodynamics, etc ...), designing ad-hoc tools for each one seems a rather inefficient way to go.

In addition to the diversity of fields of study within the smart city, there are strong constraints even in a same field. In fact, the smart city is a changing environment, with varied, nonlinear, and often impossible to predict behaviour. These various aspects of the smart city imply that to study the smart city it is necessary to be able to understand complexity. Since the smart city can be seen as a complex system, it is very difficult to correctly use the data it provides using predefined models. Rather, it seems imperative to have systems capable of learning and self-adapt to effectively process data from a complex system. So, Multi-Agent Systems (MAS) are a promising way to make best use of data collected by smart city. This kind of computerized system are indeed very well suited to perform distributed computing, and could be used to perform machine learning too.

\section{AMOEBA: A Generic AI System For SMART Cities}

In this part, we describe the generic AI java tool applied in the three next use cases, the Agnostic MOdEl Builder by self-Adaptation (AMOEBA). It builds a model in realtime using a large amounts of data from a smart city. The ability to model data behaviour offers many opportunities. A model, as we build it with AMOEBA, computes an output value using a set of input values. It could be seen as a mathematical function $f$, the set of input as vector $x$, and the output as the application $f(x)$. To be able to build this model, AMOEBA must learn using examples of correct outputs, as shown in figure 1. This put our approach in the field of supervised learning [9]. When the correct output is not known, AMOEBA could be interrogated to provide a proposition of correct output, as shown in figure 2 .

AMOEBA is a MAS using AMAS (Adaptive Multi-Agent System) approach. It is composed of agents, which are digital entities able to store information and interact with their environment. In AMOEBA, their are two kinds of agents: percept agent and context agent. Percept agents are in charge of communication with external sources of data, like sensors or database. Context agents try to define the context where a specific output would be a good one. To do that, context agents use data collected by percept agents. Whenever percepts agents receive new data, context agents use these data in order to adapt their knowledge and improve the output provided by AMOEBA.

To be able to memorize contexts where a specific output is relevant, each context agent owns a set of validity ranges. A validity range is an interval associated to one percept agent. A validity range is valid if, and only if, the current perceived value of the associated percept agent is included in its interval. If all the validity ranges of a given Context Agent are valid, the Context Agent is valid too, and its output is considered relevant. So, the memorized context where the context agent is valid could be seen as a n-orthotope (or hyperrectangle) in a space defined by all the data sources, as shown in figure 3 . The set of all current values sent by data sources could be seen as a point in this space. If this point is included in the hyperrectangle, the context agent is valid.

Each context agent also owns a local model. When valid, it asks the local model to compute an output (using the data collected by a percept agent). The local model could be implemented by any mechanism able to compute a regression. In this paper, the presented version of AMOEBA uses the Miller's updating regression algorithm[8] as local model. Using a set of points, the algorithm is able to compute a linear function. This linear function is defined by : $\sum_{n=1}^{p}\left(x_{n} v_{n}\right)+a$, with $p$ the number of percepts, $x_{n}$ a coefficient, $v_{n}$ the current value of a percept $n$, and $a$ a real number. Using various local models opens the opportunity to work on many different issues. Here we perform regression, but by changing the structure of the local model it is possible to do other things, such as classification, retaining all the other mechanisms of the approach.

An important aspect of our approach is the concept of cooperative situation. An agent is said to be in a cooperative situation if, and only if all its interactions with other agents are cooperative. An interaction between two agents is said to be cooperative if this interaction does not impair the two agents to perform their tasks. When in a cooperative situation, an agent executes its nominal behaviour to perform its tasks [4]. So, for the whole AMOEBA system to work properly, we must ensure that each agent is in a cooperative situation as often as possible, so it performs its nominal behaviour correctly. Hence, each agent must be able to :

- detect when it is in a non-cooperative situation. It is the detection step.

- adapt itself or adapt its interactions to reach a cooperative situation if needed. By doing so, it must avoid to create other non-cooperative situation for other agents. It is the resolution step.

It is through this mechanism of resolution that the agents of the system adapt themselves and, so, that the whole system adapts itself. Context agents have few different ways to adapt. They can adjust their validity range to include or exclude some values and they can change their proposed output, by adapting their local model. Moreover, new context agents can be created. It is trough these creations that AMOEBA, empty of any context agent at the beginning, is populated. Finally, context agents could remove themselves, when they consider themselves as useless.

To understand this mechanism of resolution of noncooperative situations, it is important to see how context agents adapt themselves when they are wrong. First, it is the detection step. The context agent, when valid, produces an output. If an oracle value is available, the context agent 


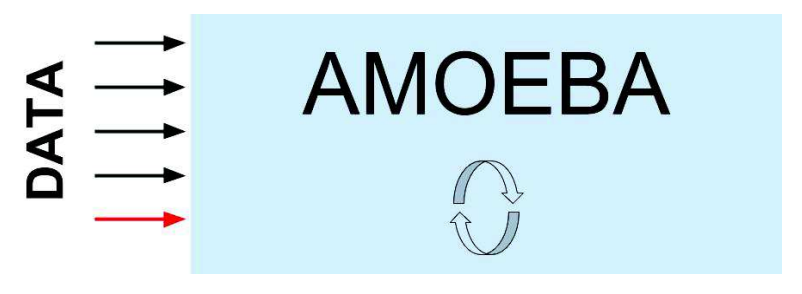

Fig. 1. During a learning step, AMOEBA uses incoming data to adapt and improve itself. Red arrow represent the specific data source that AMOEBA try to model, labeled as "oracle".

compares its output to the oracle value and, if the difference is too high (higher than an error margin dynamically determined), the context agent considers that its output was a bad one. So, it must adapt: it is the resolution step. To adapt, the agent has two options. It could change its local model to provide a different output in the same situation, and it could reduce its validity ranges to be not valid in the same situation anymore. To choose between these two possibilities, the context agent considers the difference between its output and the oracle. If very high (higher than a user parameter), the agent considers that its output is wrong in this situation, it is better to avoid to give output next time and so it reduces its validity ranges. Otherwise, it changes its local model.

Using these mechanisms, AMOEBA is able to organize itself in order to refine the outputs it offers. It was designed to be able to scale and operate in real time, thanks to the use of local interactions between agents that minimize the combinatorial explosion caused by the large amount of sources of data.

Beyond the evaluation of AMOEBA properties, there are many areas for improvements that we want to study. In particular, we are interested in opportunities to dynamically detect situations where AMOEBA lacks information to learn properly. Initially, this could raise alerts to warn that learning is ambiguous or unstable, and in a second time it could be possible to try to infer the missing information when there are correlations between them and other data sources.

Another interesting track is linked to local models of AMOEBA. We have so far worked with relatively simple local models (linear regression, fixed value, average ...), but it might be interesting to use more complex local models, such as neural networks, MAS or even other instances of AMOEBA. This would actually perform a form of metalearning, in the sense that AMOEBA would seek to determine for what situations one specific local model provides the best result.

\section{Use Case 1: Multi-Agent System for RENEWABLE ENERGY SERVICE}

Smart cities have sustainability issues especially in energy systems. Indeed, they try to reach a good quality of life providing energy services to the population such as transportation, artificial light, etc ... At the same time, they aim for

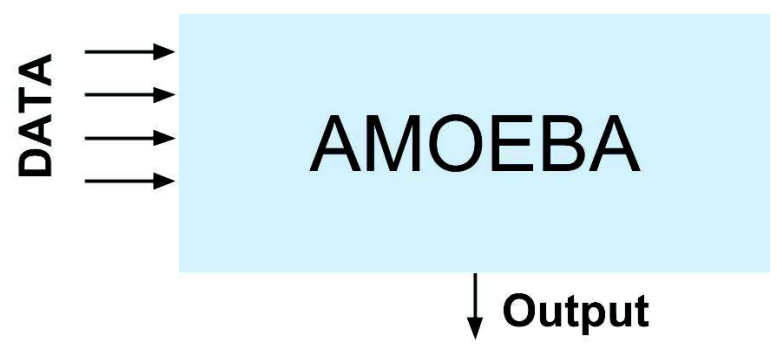

Fig. 2. During the exploitation step, AMOEBA receives a set of data without the oracle. It uses previous learning to provide an output corresponding to a possible oracle value for the current data set.

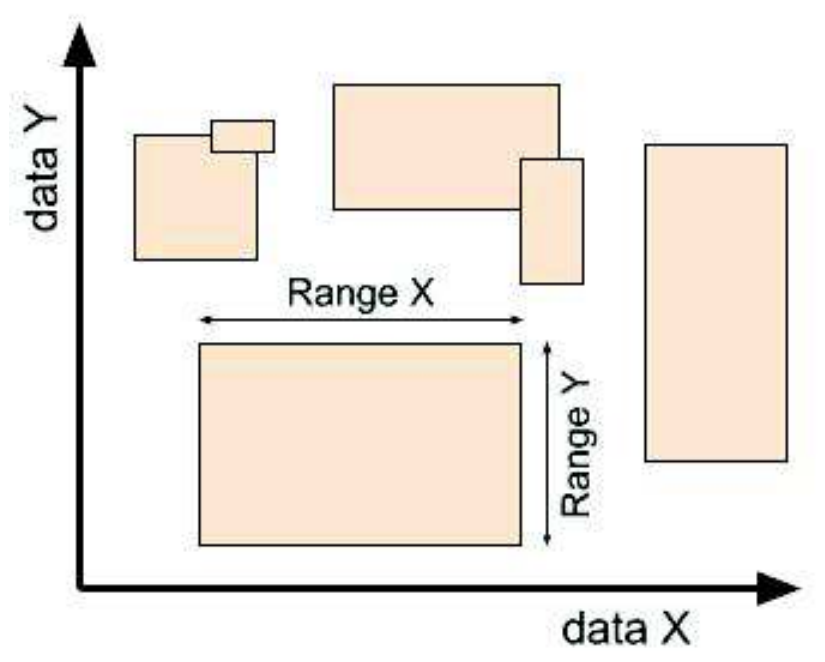

Fig. 3. Context agents could be represented as rectangles in a two dimensions space, defined by two sources of data $\mathrm{x}$ and $\mathrm{y}$. Each agent is composed of two validity ranges, which define its size.

a sustainable local economy and a reduction of the ecological impact [5].

Renewable energies like hydroelectric, wind or solar power meet these requirements. First, their sources are inexhaustible, that is to say the use of the source has no or few effects on its availability. This characteristic allows to have a return on the expensive equipment in the medium term. Then, they are distributed over the Earth and can be exploited near each city. Moreover, the ecological footprint is clearly reduced compared with fossil fuels or nuclear power. The combustion of fossil fuels is the primary source of air pollution because it releases pollutants like carbon dioxide, nitrous oxide or methane [3] . As for nuclear power, its waste is considered as high-level radioactive waste because it is potentially harmful for tens of thousands to millions of years [12].

However, the green powers are intermittent: that is to say their production of energy varies a lot over time according to climatic variations. To guarantee the balance of the electricity network (production equals to consumption) and avoid a black-out of the city, it is necessary to know the production of these types of energy at least one day in advance, so it is 
possible to start up the production of an energy source when another has a low forecast.

Furthermore, the use of renewable energies in smart cities fits into the context of fast development of the energy market. The policy of energy transition to the green growth encourages the use of renewable energy sources instead of nuclear power and fossil fuel. More and more countries in Europe liberalize their energy market adding a financial aspect in the forecast. Indeed, the producers make an offer of energy for the next day and have to respect it to avoid financial fees instead of inject it without any constraint whenever they wish.

Consequently, the forecast of renewable energy is a main challenge in order to create a smart city based on sustainable energy.

The wind power is one of the renewable energies with the most substantial progression in term of capacity added in the global consumption of electricity since 2014 [13]. It turns the force of the wind into electricity and depends upon other meteorological and mechanical parameters. The conversion of wind force into the power $P$ produced by a wind turbine follows the formula :

$$
P=\frac{1}{2} \rho S v^{3} C_{p}
$$

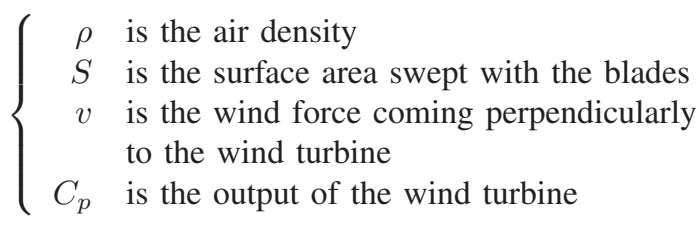

However, in reality it is harder to predict the production. For instance, the kinetic turbulence could affect the amount of wind which passes through the wind turbine. The operating speed of the blades of the wind turbine slows down when the basket temperature is too high. Another example is the presence of frost on the blades that could disrupt the mechanism. Thus, taking into account all these parameters makes the forecast of production better.

The AI system AMOEBA (presented in section III and used in this work) takes the desired number of inputs, infers their importance and combines them to give the right output which is the forecast of production in this case. To perform it, it needs historical data set of all parameters at a given instant and the associated production of the wind turbine: this system rests upon the learning over data technique.

In addition, it reduces the development time and the uncertainty compared to a more classical approach of forecast which creates a model for each meteorological phenomenon and mixes them. Indeed, AMOEBA avoids the creation of explicit models and gives a real-time forecast.

Moreover, at a given point, the wind is felt differently over time due to alteration in the environment (roughness' land, topography, albedo...) and in the measuring tool (calibration's requirement, obsolescence...). Having an adaptive tool to the environment which could detect under-efficiency (breakdowns, problem in the measuring instrument, need for maintenance...) is a great improvement for the wind power domain. Technically, AMOEBA fills the requirements to self-adapt to its environment and to provide a good forecast during the wind farm activity in spite of environmental or wind farm structure changes.

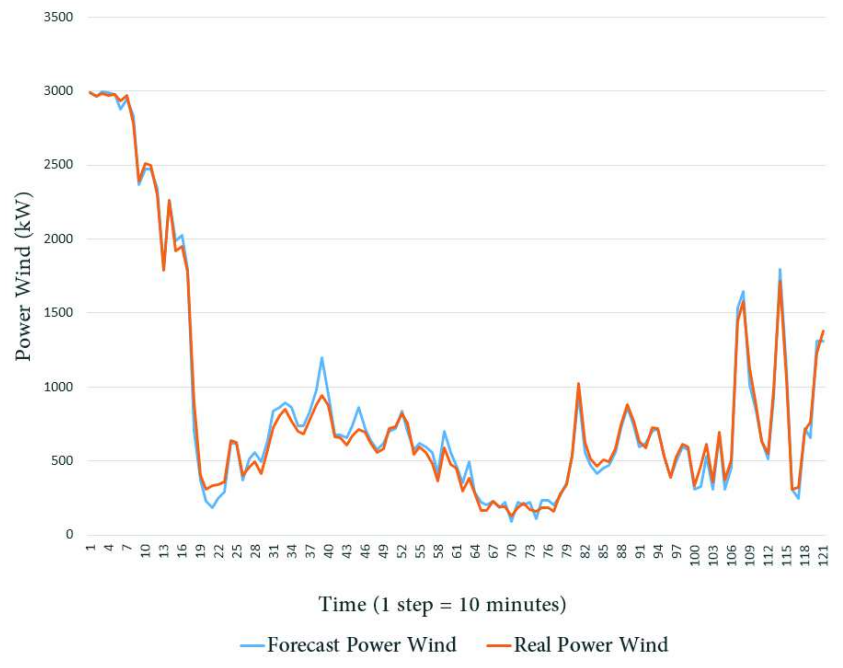

Fig. 4. Graph of the forecast provided by AMOEBA and the real wind power of a wind turbine of the wind farm in France during more than 20 hours. The historical data set for learning and resolution steps is made with data of the force of the wind at the basket of the wind turbine and the corresponding production from 2015 .

The forecast made by AMOEBA and the real production of a wind turbine in the wind farm, situated in France, over more than 120 steps of resolution is presented in figure 4 . This forecast has linked the force of the wind received at the wind turbine and the wind power produced. The normalized root-mean-square deviation or error (NRMSD or NRMSE) is $1.92 \%$ and the normalized mean absolute error (NMAE) is $1.44 \%$. The first one indicates there is few unlikely errors of forecast whereas the last one shows the constant error made during the resolution is low.

Finally, AMOEBA is promising for treating forecast problem with real data. But the one of energy production relies on the meteorological forecasts in order to be used in advance. That leads to some difficulties for the resolution as the meteorological forecasts bring inconsistent data to learn with. Indeed, the error of the meteorological model generates ambiguous situation during the learning step: two similar forecasts can give two really different productions. This problem can be explained on one hand because the production of wind power depends upon a lot of parameters, as shown in the formula above, and each forecast of a parameter adds its uncertainty. On the other hand, the meteorological forecasts are made every hour by predicting the conditions of the last ten minutes of the hour. So, one hour is explained with a ten minutes forecast. The last but not least, these forecasts are provided at points of a defined mesh which aren't the location of the wind turbine ; the characteristics of the area are different between them and cause differences 
in the meteorological conditions. Some effects like the wake effect of the wind turbine can also come under consideration.

To anticipate this problem, it should be interesting to find some patterns where the differences between the forecast and the real condition are recurrent. For instance, when the density is high and the kinetic turbulence is low, the forecasts tends to be under-estimated. At the current state, AMOEBA isn't sufficiently appropriate to perform this recognition.

Forecasting the production enables to use different sorts of renewable energies. So, this kind of tool is necessary to manage efficiently the energy in a smart and sustainable city.

\section{Use Case 2: Multi-Agent System for Economy ENERGY}

Nowadays, a lot of objects are connected like our watches which can receive messages, the refrigerators which can order missing products, or the parkings able to indicate free places. It is easy to think that buildings will be connected in the cities of future. They will dispose of sensors to measure their water consumption or their electric consumption. With those data, it will be possible to detect uncommon situations, that we named singularities. The ability to detect these singularities could improve a building consumption and so saving energy.

For example, if the singularity is a breakdown, a technician can react to it faster and, consequently, the energy management will be improved. An efficient management of energy is important for the smart cities [11]. We will be able to go for the way off, we can make a comparison between the sensors data and the building simulation. This also offer promising opportunities to improve study of user behaviour on energy consumption, by differentiating more precisely measurement errors and unexpected user actions for example. In some project (like the zero energy building project [1]), understanding energy consumption with precision is an utmost necessity.

During the experiences, we have used the data from an already connected building, and more precisely the data of the handling air central. AMOEBA was designed to learn to correlate data. Here, we use it to make classification. We use five sensors as entry, and the oracle is a sixth entry saying if the current situation is a singularity or not. Then, AMOEBA use these entries to build a more general model of singularities in such a way that, in a new unknown situation, AMOEBA could said if this situation is a singularity or not.

- One sensor for the outside air temperature.

- One sensor for the temperature ambient air, in other words it is the temperature in the life area.

- One sensor for the temperature resumption air. It is the air temperature on the entrance of the central air handling.

- One sensor for the temperature blown air. It is the air temperature to send back in the building.

- Finally, the sensor for the blown pressure. When this

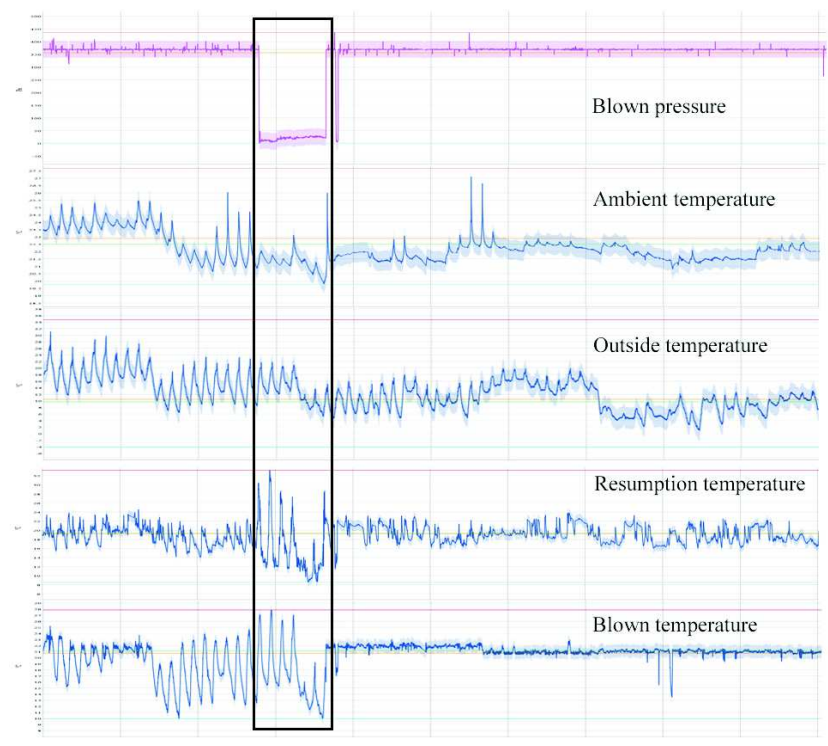

Fig. 5. The period is between october 12 and december 21 2014. The pressure is between 0 and $450 \mathrm{~Pa}$. The ambient temperature is between 19 and $28 \mathrm{C}$. The outside temperature is between 1 and $31 \mathrm{C}$. The discard temperature is between 9 and $33 \mathrm{C}$. The blown temperature is between 10 and $28 \mathrm{C}$.

sensor value is too high or too low, we have a singularity.

This chart figure 5 represents the five sensors values, it covers the period from october 12th to december 21th 2014. A significant singularity is also visible on the chart because it is on several temperatures sensors of the resumption air, the blowing air and on the pressure sensor.

After the learning step over the 38,809 situations (see Figure 6), AMOEBA has well classified 38,314 situations. This represents $98.7 \%$ of success. It has mistaken 495 situations and 449 singularities were not detected.

AMOEBA succeeds at classifying the major singularity but it does not succeed to classify all singularities. On 2352 singularities, AMOEBA has well classified 1903 situations which corresponds to an error rate of $19 \%$.

In order to improve those results, AMOEBA has to improve the generalization of the situation but it is difficult because with five sensors, the generalization is on five dimensions We have to lead further experiences with others parameters to confirm those results. For example, we can increase the number of sensors to better classify the situations or asking to an expert to annotate the situations. But the problem of generalization goes further than that. Indeed, determining a good way to generalize is a complex task. In the current version of AMOEBA, results could be modified by tuning parameters affecting the generalization process. Even if this tuning is rather simple for a learning system, we are working on a more general way to generalize, able to be fully efficient without tuning.

This kind of systems serves the economy of energy and is needed to help human to manage larger and larger systems. 


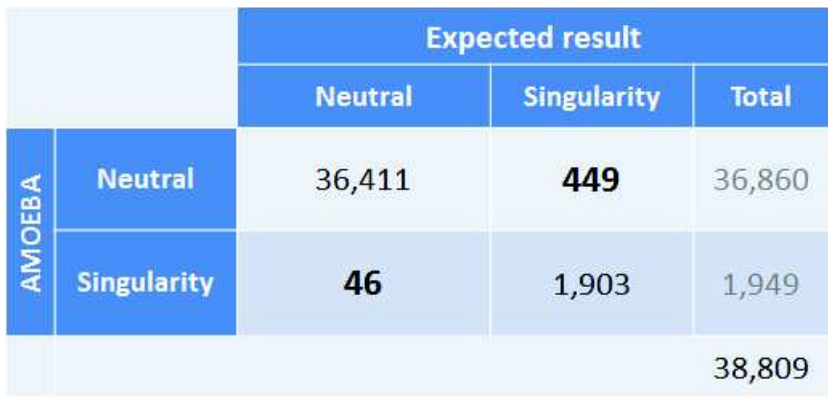

Fig. 6. AMOEBA output according to expected output (neutral or singularity)

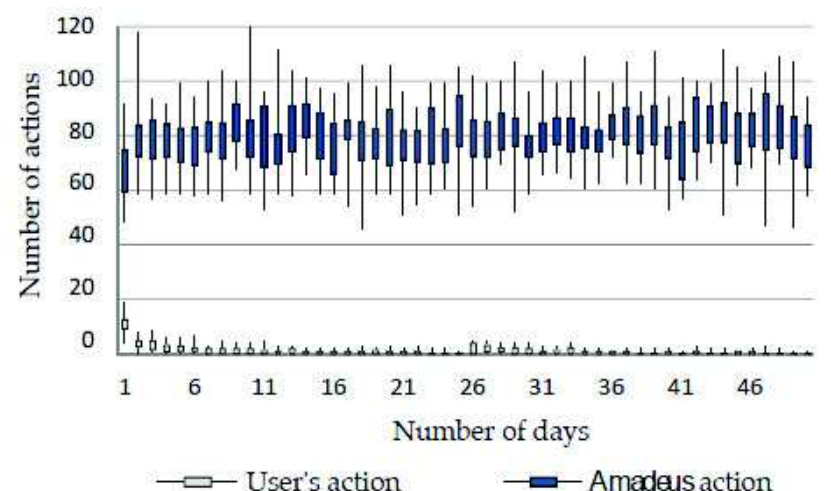

Fig. 7. Actions on devices: Autonomous AI tool versus human

\section{Use Case 3: Multi-Agent Systems for MANAGING THE WELL-BEING}

A previous work [6] demonstrated the capability to observe users during their daily activity in order to learn how to perform their actions on their behalf. An example of this work is given on the figure 7 which simulates the human behaviour during 50 days in a room of a flat having a light and an electric shutter. We observe that the AI system learns relevantly the contexts in which the user acts: on average 78.6 actions by day while the user performs only 0.9 action by day. Because a human has not exactly the same behaviour, the simulations are repeated 20 times during the 50 days period. We want now to go a step beyond, in learning what is the real underlying well-being of the humans and not only their actions on the devices.

However, this tool doesn't express the human needs because it only informs about his actions on the devices in numerous contexts. So, it is important to have means to perceive human requirements and means to act on the environment in order to maintain the well-being which can evolve in real time and which is not the same for two different inhabitants. The sensors and effectors must be embedded with intelligence to enable all the system to selfadapt to the changes.

The well-being is defined as a pleasant state resulting from the satisfaction of the body and the spirit requirements which leads to feel a so-called sensation of well-being. This satisfaction towards the environment rests on all the physical dimensions of the atmospheres, but also to the behavioural and psychological aspects. At the physical level, or physiological, there are the comforts which can be measurable thanks to sensors such as the respiratory, thermal, acoustic or visual comforts. This aspect is generally well-known and several norms define minimum and/or maximum thresholds for concerned physical quantities (light, temperature, acoustic power, etc.). At the behavioural level, the individual human capacity for action is highlighted because the environmental conditions and the expectations vary over time. Indeed, a draft trough a room is more appreciate in summer than in winter. Thus, it is important that the occupant can act on the regulatory bodies of the systems modifying the conditions of his environment. Finally, at the psychological level, the implication of the individual is pointed out in term of energy. Despite of his capacity to control his environment to meet these physiological needs, he has to be conscious of it. There are other dimensions to the sensation of well-being in an environment. For instance, the comfort of use (is the building adapted to the activities for which it is designed?), the aesthetic feeling (is the building beautiful? Does it well integrate to his environment?), a positive or negative feeling due to the innovation (is the building too modern?), etc. So, the combination of the various comfort dimensions (physiological, behavioural, psychological) involves the well-being in an environment isn't easy to describe. This well-being will be not only different for each person, but also time-varying, according to its age, its sex, its health, and even its humor.

The ecosystem of smart city aims to study this notion in order to improve the comfort of the citizens and to be more effective, while developing the city in accordance with the environment.

Some studies on the respiratory, thermal, acoustic and visual comforts were made, but in such a divided way, without studying the interactions between them. In this work, we would like to take simultaneously into account all these comforts with an "adaptive" and "bottom-up" approach. A cyberphysical system is associated to the management of one type of comfort, for example for visual comfort. This system has some information in inputs, information provided by sensors. The outputs of the system is realized by effectors which act on the comfort. The system able to manage all the types of comfort is a collective of cyberphysical systems or a system of systems. All these systems are in interactions and interdependent.

AMOEBA makes a learning of the various systems linked to the comforts, thanks to MAS, without describing them the intrinsic features of the comforts to learn. Each agent possesses its own objective which could conflict with the others. Like this, a conflict between visual comfort and thermal comfort could occur during a beautiful summer day between the actions of opening and closing the shutters. Indeed, if an action of closure increases the thermal comfort by decreasing of the temperature, it will be made to the detriment of the visual comfort ; the action of opening will cause the opposite effect. Furthermore, if the decrease of the 


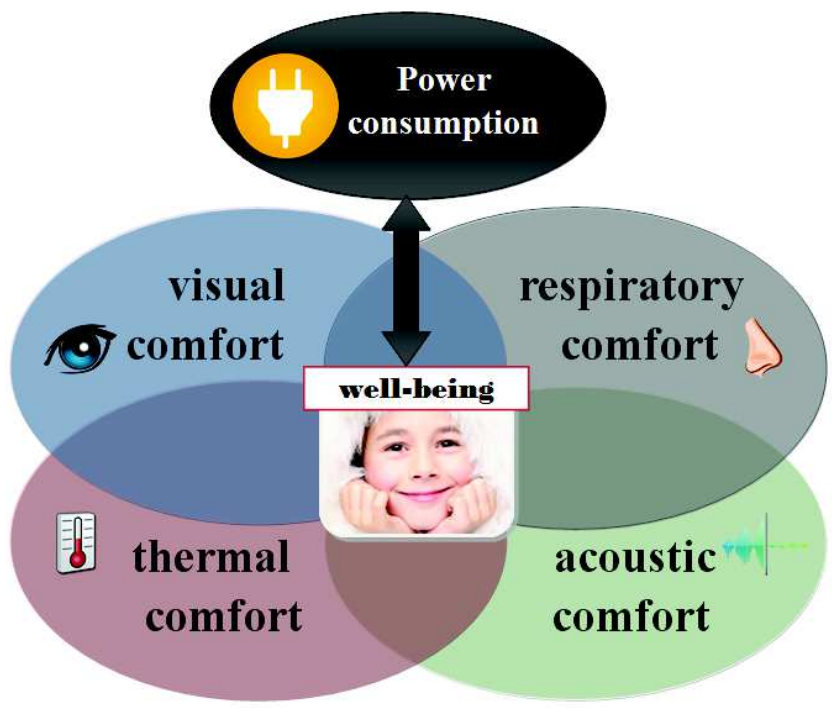

Fig. 8. The respiratory, thermal, acoustic and visual comforts define the concept of well-being and interact on power consumption.

visual comfort engenders the simultaneous action to switch on a light, the MAS bound to the electric consumption will conflict with this action. It is this peculiarity which allows the emergence of features and actions, and which can reveal the notion of well-being studied.

Because in smart and sustainable system the human is central, the system must be able to take into account and to act to provide them a good quality of life.

\section{CONCLUSIONS}

Smart cities are complex environments. In this paper, we emphasize the importance of considering the management of a smart city as a complex multi-scale problem, requiring a generic and adaptive approach. Through three use cases, dealing with the energy production, the singularity detection and the comfort, we present our multi-agent and bottom-up approach to address these requirements. This multi-agent approach is based on a set of cooperation mechanisms, defined by the AMAS approach, to ensure its rapid adaptation to the changing dynamics of a complex environment.

We presented 3 use cases of a generic system AMOEBA able to learn and to self-adapt to changing environment. The first application deals with the renewable energy where the system provides a forecast of the production. The second application concerns the detection of singularity or singular behaviour in order to help to repair these situations to economize energy. The third is a system of systems able to manage the daily life environment of human in order to provide them well-being. In these three use cases the generic AMOEBA system is instantiated.

AMOEBA is able to design in a bottom-up approach a system which can present a kind of intelligent behaviour because it learns from the encountered situations. These systems are pervasive in the sense that they can work without the human intervention if the human doesn't intervene. And they take into account it if he human acts on the system.

In the smart and sustainable city, these kinds of systems are needed because of the complexity of the city where all situations cannot be forecast in advance.

The various use cases presented will have to be refined and further developed in the future. In the medium term, our approach will be soon tested in the context of a connected campus with a very large amount of data, as part of the neOCampus operation. This should assess, hopefully, the capacity of our approach to process data from a system of complexity approaching the complexity of a smart city.

\section{ACKNOWLEDGMENT}

This work is funded by the University of Toulouse III - Paul Sabatier and the Midi-Pyrénées region. It is a part of neOCampus, a scientific and interdisciplinary operation focusing on the development of a connected, smart and sustainable campus.

A part of this work has been made in collaboration with the wind power forecasting company *SWIFT.

\section{REFERENCES}

[1] Kylili Angeliki and Fokaides Paris, A. European smart cities: The role of zero energy buildings. Sustainable Cities and Society, 15:86-95, 2015.

[2] Michael Batty, Kay W Axhausen, Fosca Giannotti, Alexei Pozdnoukhov, Armando Bazzani, Monica Wachowicz, Georgios Ouzounis, and Yuval Portugali. Smart cities of the future. The European Physical Journal Special Topics, 214(1):481-518, 2012.

[3] Fatih Birol and al. Energy and climate change, 2015.

[4] Noelie Bonjean, Wafa Mefteh, Marie-Pierre Gleizes, Christine Maurel, and Frédéric Migeon. Adelfe 2.0. In Handbook on Agent-Oriented Design Processes, pages 19-63. Springer, 2014.

[5] Eduardo de Oliveira Fernandes Fernandez, Leonardo Meeus, Vitor Leal, Isabel Azevedo, Erik Delarue, and Jean-Michel Glachant. How to foster a quick transition towards local sustainable energy systems, 2011.

[6] Valérian Guivarch, Valérie Camps, André Péninou, and Pierre Glize. Self-adaptation of a learnt behaviour by detecting and by managing user's implicit contradictions. In Proceedings of the 2014 IEEE/WIC/ACM International Joint Conferences on Web Intelligence (WI) and Intelligent Agent Technologies (IAT)-Volume 03, pages 2431. IEEE Computer Society, 2014.

[7] Rob Kitchin. The real-time city? big data and smart urbanism. GeoJournal, 79(1):1-14, 2014.

[8] Alan J Miller. Algorithm as 274: Least squares routines to supplement those of gentleman. Applied Statistics, pages 458-478, 1992.

[9] Mehryar Mohri, Afshin Rostamizadeh, and Ameet Talwalkar. Foundations of machine learning. MIT press, 2012.

[10] Taewoo Nam and Theresa A Pardo. Conceptualizing smart city with dimensions of technology, people, and institutions. In Proceedings of the 12th Annual International Digital Government Research Conference: Digital Government Innovation in Challenging Times, pages 282-291. ACM, 2011.

[11] Rocha Paula, Siddiqui Afzal, and Stadler Michael. Improving energy efficiency via smart building energy management systems: A comparison with policy measures. Energy and Buildings, 88:203-213, 2015.

[12] K.R. Rao. Radioactive waste: The problem and its management. Current Science, 81(12):1534-1539, 2001.

[13] Janet L. Sawin and al. Renewables 2015 global status report. Technical report, Renewable Energy Policy Network for the 21st century, 2015.

[14] Xindong Wu, Xingquan Zhu, Gong-Qing Wu, and Wei Ding. Data mining with big data. Knowledge and Data Engineering, IEEE Transactions on, 26(1):97-107, 2014. 